EPJ Web of Conferences 92,02097 (2015)

DOI: $10.1051 /$ epjconf/ 20159202097

(C) Owned by the authors, published by EDP Sciences, 2015

\title{
Proposal for an electro/fluidic no-moving-part transducer based on wall-jet separation from a heated curved surface
}

\author{
Václav Tesar̆ \\ Institute of Thermomechanics v.v.i., Academy of Sciences of the Czech Republic \\ Dolejškova 5, 18200 Praha-Kobylisy, Czech Republic
}

\begin{abstract}
Transducers for varying output flow from a fluidic device in dependence on electric input signal are increasing in importance - and yet no their design has been so far fully satisfactory. Except those that handle very extraordinary liquids (e.g. electro-rheologic), the transducers operate in two stages. The first stage is conversion into a motion or deformation of a mechanical component. In the second stage this mechanical effect acts on the output fluid flow. This signal conversion involving mechanical movements is a weak link between no-moving-parts electronics as well as no-moving-parts fluidics. Mechanical components complicate manufacturing, have tendency to get stuck or become worn - or, if deformed, may break (e.g. due to material fatigue). Their inertia limits the frequency range. Author here proposes a new transducer idea. The electric input heats the wall to which is attached a fluid jet, causing separation of the jet from the surface. Preliminary experiments show that relatively small heating suffices to change the flowfield substantially — and the reduction of the attachment wall to a thin metal foil can make the frequency range quite acceptable.
\end{abstract}

\section{Introduction}

Fluidics handles fluids and controls their flows. Classical approach to these tasks has been mechano/fluidic: the handled fluid was acted upon by a mechanical component in the device. Pure fluidics does not use mechanical moving components and this brings useful advantages. Increasingly often, the fluid handling systems are nowadays controlled by electronic controllers - and this calls for availability of electro/fluidic signal transducer. Since there moving components are absent in semiconductor electronics as well as in fluidics, it is evident that also the transducer should not have any mechanical components - to stop being the weak link between the electronic and fluidic systems.

A no-moving-part solution of the E/F (electro/fluidic) conversion became recently particularly important because of two current developments (a) One them is the rapidly growing field of microfluidics [1], often with microelectronic circuits made on the very same substrate as the cavities for fluid flow. (b) The other reason is the recent increasing use of fluidic oscillators as means for improving efficiency of various processes such as, e.g., heat and mass transfer, which benefits from the agitation [2], or the generation of microbubbles [3] where the oscillation avoids bubble growth by conjunctions [18]. Although the key selling factor of the fluidic oscillators is their autonomy and simplicity (for reliability and low cost), even there is now often requested a control by electronics.
Existing electric control of fluid flow in fluidics remains unsatisfactorily due to absence of a direct electric action on typical working fluids, such as air and water, which are electrically neutral. Contemporary transducing devices have therefore to resort to an indirect two-stage mechano/fluidic conversion. The most widespread approach is electrically generated mechanical motion of a flap or similar steering component protruding into the fluid filled volume as the first stage - and then, in the subsequent second stage, a mechanical action of this steering component on the fluid.

The desirable ideal of no-moving-part conversion is only realisable with very special working fluids such as, e.g., those exhibiting electro-rheologic effects [4], magneto-rheologic phenomena [5], or liquids containing colloidal ferrite $[6,7]$. This special-fluid approach is, however, only rarely acceptable, because the fluid in a fluidic system cannot be chosen arbitrarily. Its choice is usually dictated on one hand by availability in the system and on the other hand by the requirements of end use. The latter limitation is obvious if the task the fluidic system performs is an analysis of a fluid sample. Another obvious example is the case of operating the fluidic oscillator with $\mathrm{CO}_{2}$ in an algae growing bioreactor [3] where, of course, no electrically active fluids can replace the role of $\mathrm{CO}_{2}$ in algal photosynthesis.

The presently used two stage E/M/F electro/mechano/fluidic conversion with the intermediate mechanical action is unsatisfactory from many points of view. In fact the pure fluidics, with fluid flows in

\footnotetext{
a Corresponding author: tesar@it.cas.cz
} 
constant-geometry cavities, was developed with just the intention to eliminate the drawbacks caused by the presence of mechanical components. As far as microfluidics is concerned, perhaps the most troublesome aspect of the presence of moving parts is the manufacturing complexity that they make necessary. The moving components have to be manufactured separately and placed into their locations, which calls for expensive assembly operation. Another problem is the generally decreased reliability and limited working life due the possibility of the moving parts getting stuck or worn and hence losing their precise shape. Also, the movable components do not work properly when exposed to acceleration (vibration, shocks), especially with the present-day fluidics tending to operate with low fluid pressure levels and hence moving the mechanical components by relatively small forces compared to their inertia. Of course, last but not least reason is the inertia of mechanical components, which limits the achievable speed of response to input.

Some troubles associated with the movable components may be removed if their translating or rotating motion is replaced by elastic deformation. This, however, is also far from ideal solution. Apart from the limited extent of the motion there is the problem of typical base plate materials not possessing sufficient deformability. Thus the flexible parts also require being made separately, calling for the undesirable assembly operations in the manufacturing processes. Another, perhaps even more important problem is the material fatigue of the deformed components. This is likely to limit reliability and working life of the fluidic device.

Also the generation of the mechanical motion is not without its problems. Quite usual approach is the firststage signal conversion by an electromagnet. Manufacturing its winding from a length of insulated wire is certainly a process alien to the principle of the single-step manufacturing by photoetching in microfluidics. In fact, practical electromagnets usually tend to be inconveniently large for microfluidic size scale. They have thus to be made separately and then

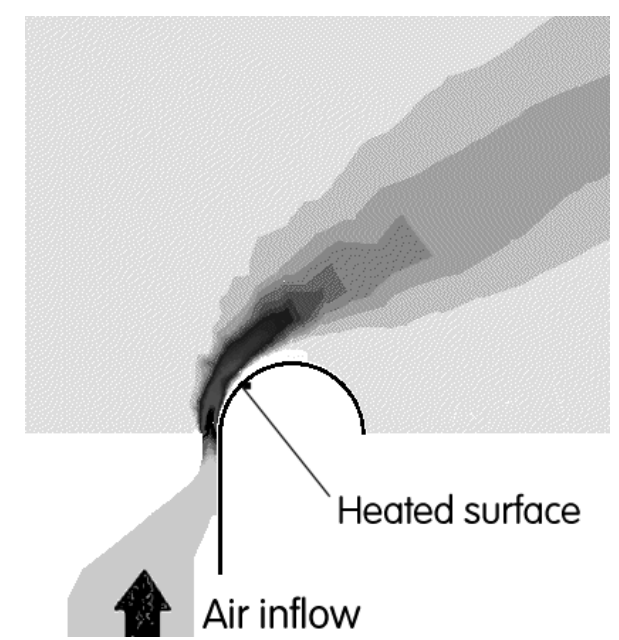

Figure 1. The basic principle of the discussed electro/fluidic transducer is flow separation of a wall-jet caused by heating the attachment wall (computation result from [12]). placed and fixed into their positions, again at the added cost of the assembly operation.

Many of these unpleasant or inconvenient aspects are also shared by the other currently popular application area of fluidics, the oscillators in processes intensification. Apart from sharing the shortcomings mentioned above (like the limited reliability), the main draw-back of the movable or deformable components in this application is the limitation they place - as the consequence of their inertia - on the upper end of the range of operating frequencies.

It is obvious that a considerable freedom from all the above listed problems would result if it were possible to develop an electro/fluidic signal conversion method within fixed-geometry cavities. The present paper discusses one of the very rare working principles that does not need any special electro-active working fluid. Admittedly, this conversion is also of two-stage character, but the mechanical motion as the intermediate step is replaced by thermal effects. This is not a wholly new idea, apparently being already patented [8] as long ago as in 1971. Nevertheless it has not been pursued to a use in a practical, manufactured device - very probably because there was the obvious fear of slow response, generally associated with thermal effects. Another factor that made it perhaps not attractive was the expected necessary high electric current control signal levels.

\section{Feasibility experiment}

An interesting but little known aerodynamic phenomenon with a quite close kinship to the principles employed in jet-type fluidic devices (the jet attachment, known as Coanda effect, is widely used in fluidics) is separation of a wall-jet flow from its wall if the latter is heated. The heating is quite easily achieved electrically, the simplest method being Joule heating by the electric input signal passing through the attachment wall made of conductive material. The reason why it has been so far ignored for use in transducers is the general experience of slowness of thermal phenomena. This, however, is due to the usually large thermal capacities of typical relatively massive and voluminous heated objects. The problem may be solved by reducing the size of the attachment wall so that it is just a very thin metal foil. Since in its role of an attachment wall this foil is exposed to the flow of cool fluid, not only the heating but also the cooling action taking place after the termination of heating is likely to be quite fast.

Provided the question of thermal inertia is answered, the thermal-effect approach offers several advantages. The transducer may be very simple and small. It has no moving parts (and hence its response is not limited by their inertia). It may be manufactured essentially by the standard manufacturing steps common in fluidics, perhaps together in a single manufacturing step with other fluidic components. The configuration of the fluidic side of the transducer may be identical to the that of standard fluidic jet-type flow-diverter amplifiers. This bring as an additional advantage easy impedance matching [17] with other devices in fluidic circuits. 
Since the limitations of upper end of frequency range by thermal effects in thin walls are difficult to predict, it was decided to perform an experimental feasibility study. As the starting point were used the earlier results described in [11] and [12], where the heating was oriented to study a different problem stability of shear flows in the presence of strong temperature gradients. Another helpful source of indoemation were results of experiments with heated thin wire passing through the axis of an axisymmetric jet, as described in [10]. They demonstrated convincingly the capability to cause quite dramatic flow control effects if the heating leads to a change in transition from laminar flow to turbulence. The associate low Reynolds numbers were not considered a disadvantage, especially for the use in microfluidics.

Unfortunately, very little information could be found by search in existing literature. It revealed only the patent [8] and reference [9], which is no more accessible. Thus the proposal discussed here is based on the configurations from [11] and [12] using the author's simple laboratory setup from that time. Its geometry is two-dimensional planar, with the space for air flow between two parallel end-plates (made of transparent PMMA, Fig.3). The plates were separated by the constant distance $\mathrm{h}$ between them. The air was supplied into a rectangular nozzle of dimensions $h \times b$ where the width $b$ was adjustable. At one side of the nozzle is clamped one end of the metal foil, connected to one terminal of the electric current source. The foil continues upwards reaching above the nozzle exit, where it is curved at a constant curvature radius $r$. Originally the curvature ended at an angular distance $\boldsymbol{N}=\mathbf{2} \pi / \mathbf{3}$ from the nozzle exit, from where it continued straight to the other clamp, which is connected to the other current source terminal. In the photograph of the test rig in Fig. 3 may be noted a dial gauge indicator used to measure the

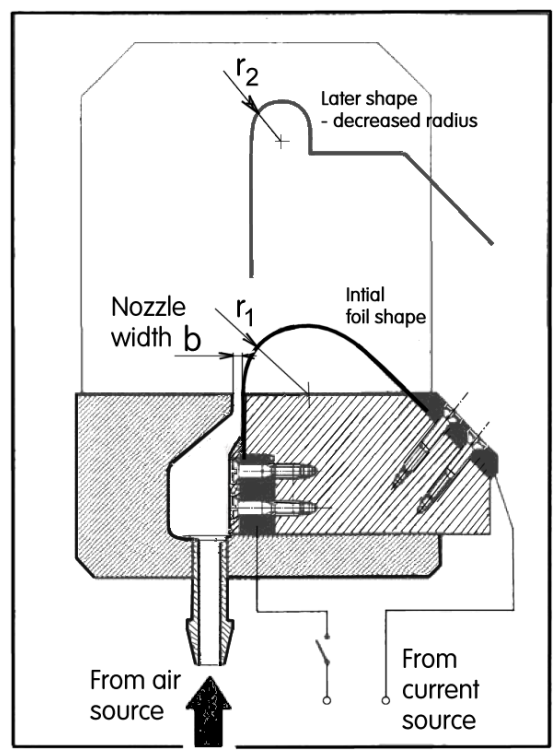

Figure 2. Layout of the laboratory model. High frequency range was obtained by reducing the attachment wall to a thin foil, Joule-heated by the electric current passing through it [11]. In later phase of tests, the range of Reynolds numbers was increased by deforming the foil (as shown in top part of the picture) to obtain smaller curvature radius $r_{2}$.

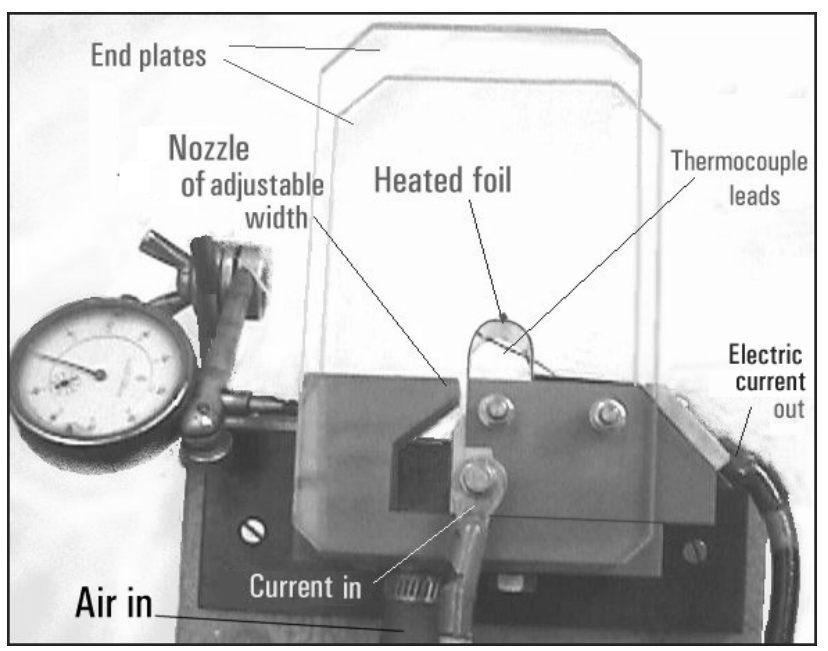

Figure 3. Photograph of the experiment used to detect the thermal dependence of attachment. Note the later foil shape (Fig. 2). The dial indicator shows the adjusted nozzle width $b$.

adjusted nozzle width $\mathrm{b}$ with precision to $0.01 \mathrm{~mm}$ ). Experience has later shown desirability of a smaller curvature radius of the foil. This was obtained, decreased from the original $r_{1}$ to $r_{2}$ (Figs. 2, 3), by suitable deformation of the foil. Experiments demonstrated convincingly that the character of the flow is determined uniquely by the ratio $\mathrm{r} / \mathrm{b}$.

With the flowfield geometry adjusted, the temperature $T_{w}$ of the foil, measured by a thermocouple (visible Fig. 3), was also set. Starting regime was one with the jet attached to the foil. Then the flow rate, measured by a flowmeter, was gradually decreased, watching the value at which the wall-jet separated from the foil. For a particular geometry $\mathrm{r} / \mathrm{b}$ this critical state of separation was found to be uniquely dependent on the Reynolds number

$$
\operatorname{Re}_{\mathrm{b}}=\frac{\dot{\mathrm{M}} \mathrm{V}}{\mathrm{h} \nu}
$$

- where $\dot{M}[\mathrm{~kg} / \mathrm{s}]$ is the measured mass flow rate, $v$ $\left[\mathrm{m}^{3} / \mathrm{kg}\right]$ is the specific volume of air, and $v\left[\mathrm{~m}^{2} / \mathrm{s}\right]$ its (kinematic) viscosity.

The critical separation states were also found for flows with cold (i.e., not heated) foil. The results of the measurements are presented in Fig. 4. A remarkable fact immediately seen there is the sensitivity of the flow separation effect. Even an increase in temperature by mere $20 \mathrm{~K}$ was sufficient to cause a substantial change in the character of the investigated flowfield.

\section{Enigma of high sensitivity}

Of course, the investigated separation effect is due to the change in air viscosity. However, the changes in the critical values in Fig. 4 are much larger than what could be simply explained by the corresponding change in Reynolds number.

In the regimes with the electric current switched on it is necessary do discriminate between the nozzle exit Reynolds number $\mathrm{Re}_{\mathbf{b}}$ defined by eq. (1) and the local 


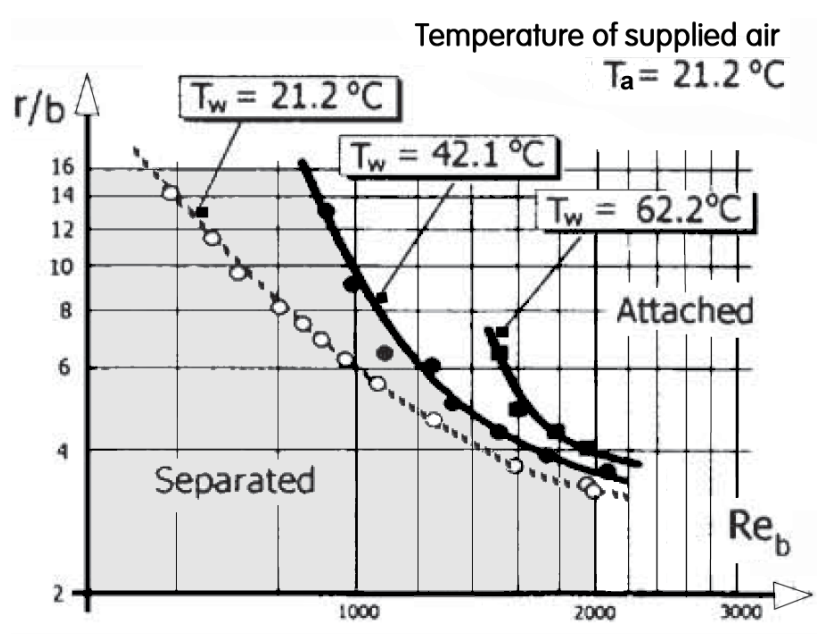

Figure 4. Experimentally detected boundaries between the attached and separated flows past the curved foil ( $r$ is curvature radius) at different foil temperatures $T_{w}$. Reynolds number $R_{e}$ is computed for the conditions in the supply nozzle (where it is not influenced by the heating).

Reynolds number $\mathrm{Re}_{\text {local }}$ of the flow at the heated attachment surface. The latter was not accessible for measurement and direct evaluation, but it is obvious that it was decrease when the foil was heated. To return the flow to the same conditions necessitated an increase in the cold flow $\mathrm{Re}_{\mathrm{b}}$ plotted on the horizontal co-ordinate in Fig. 4. This increase in $\mathrm{Re}_{\mathbf{b}}$ at which the switching occurred explains the inclination and curvature of the lines connecting in Fig. 4 the data saymbols.

The shift of the data points to the right in Fig. 4 at the same $r / b$ with increase of foil temperature from $T_{w}$ $=21.2{ }^{\circ} \mathrm{C}$ to $\mathrm{T}_{\mathrm{w}}=42.1{ }^{\circ} \mathrm{C}$ should be now noted. It is remarkably large considering the relatively small temperature increase. Since the thermal dependence of viscosity of air is known, it is possible to compute the theoretical shift of the critical boundary.

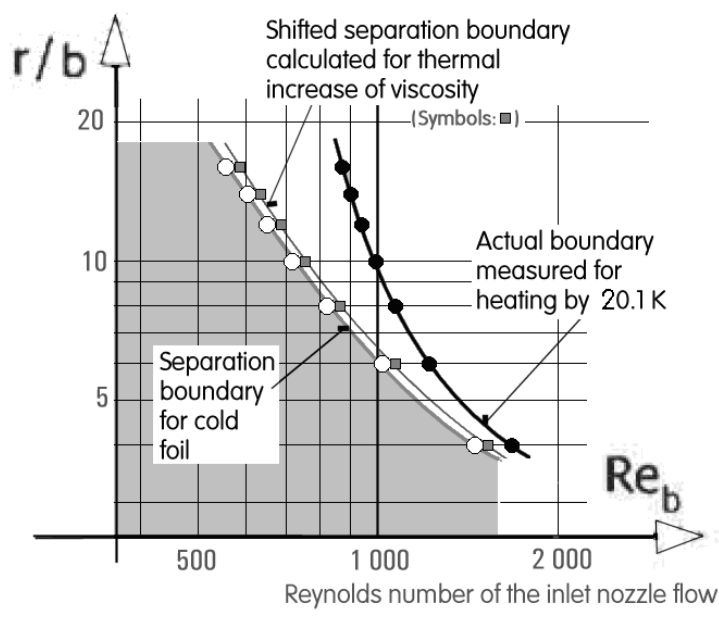

Fiure 5. Enigma of experimental results. Increasing the foil temperature by $20.1 \mathrm{~K}$ increases viscosity by an amount which cannot explain the experimentally found large shift of the switching boundary by small heating - the effect on which the proposed transducer is to be based.
A good approximation of the viscosity vs. temperature dependence is provided by the relation

$$
v=1.456 \times 10^{-6} \mathrm{~T}_{\mathrm{w}}^{1.5} \mathrm{v} /\left(\mathrm{T}_{\mathrm{w}}+110\right)
$$

At the temperature $\mathrm{T}_{\mathbf{w}}=21.2{ }^{\circ} \mathrm{C}$ of the non-heated flow in Fig. 4 the viscosity according to eq.(2) is

$$
\boldsymbol{v}=15.2 \times 10^{-6} \mathrm{~m}^{2} / \mathrm{s}
$$

Obviously, this fails as an explanation of the observed sdata points shift effect. By heating the foil to $T_{w}=$ $42.1^{\circ} \mathrm{C}$ the viscosity increases only 1.045 -times to the value

$$
\boldsymbol{v}=16.0 \times 10^{-6} \mathrm{~m}^{2} / \mathrm{s}
$$

The corresponding shift of Reynolds number values $\operatorname{Re}_{\mathbf{b}}$ to the right-hand side in Fig. 4 at the same r/b due to the change from eq. (3) to eq. (4) is so small that in Fig. 5, when it is plotted, the data symbols $\square$ are shifted very little. In fact the shift is as small as to be almost difficult to discriminate from the round symbols for the cold flow regime. It is obvious that the change - such as e.g. from the value in eq.(3) to that in eq.(4) - cannot explain the observed actual shift of the critical switching line in Fig. 4. With this small effective increase of Reynolds number $\mathrm{Re}_{\mathrm{b}}$ caused by heating, the transducer could not be reasonably operated.

\section{Explanation: Separation bubble}

The enigma of the much larger than expected response to the heating of the attachment wall may be quite convincingly explained by considering phenomena that take place in separation bubbles - known to occur at the leading edge of aerofoils [13]. They are likely to be also present here, under the wall-jet attached to a curved wall at low Reynolds numbers. In the regime of the attached wall-jet the bubble is closed - as shown schematically in Fig. 6. The closure is due to the transition into turbulence. At Reynolds numbers typical for the transducer operation the flow upstream from the bubble, in the regime A, Fig. 6, is laminar. As such it is likely because of only very weak entrainment - to separate from the attachment wall at a rather small angular distance $\boldsymbol{N}$ from the nozzle exit. In the schematic

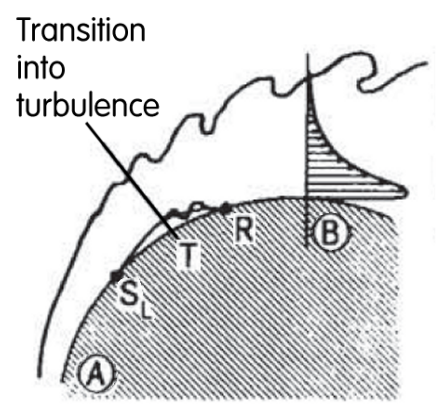

Figure 6. Author's hypothesis explaining the attachment at low Reynolds numbers. In the cold flow regime the wall-jet detaches from the surface at $S_{L}$,. The absence of stabilising effect of the wall causes transition into turbulence in $T$. This is associated with rapid growth of jet thickness and results in reattachment (in R) so that the flow remains attached. 


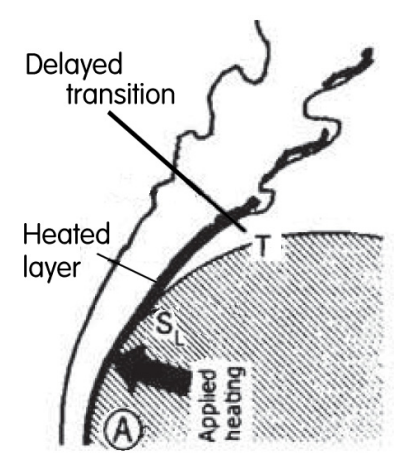

Figure 7. If the thin near-wall layer is heated by heat transfer from the wall, transition is delayed and the near-wall layer remains laminar. No re-attachment occurs and the flow separates at a short angular distance $\curvearrowright$ from the nozzle exit.

presentation of Fig. 6 the separation takes place in the point $S_{L}$. Once the jet separates, it is no more affected by the stabilising influence of the wall. As a result, the transition into turbulence occurs in the location $\mathrm{T}$. Characteristic property of turbulent shear regions is their rapid growth of thickness. In the case of Fig. 6 this growth is so rapid that the inner side of the jet (i.e. the near-wall side) soon reaches up to the attachment wall. It re-attaches there in the location R. Downstream from it, in the regime (B), the flow is turbulent in spite of the perhaps rather low nozzle exit Reynolds number $\operatorname{Re}_{\mathrm{b}}$.

The turbulent entrainment causes sufficient pressure difference between both sides of the jet to keep it attached.

The next Fig. 7 presents, again schematically, the conditions at the heated attachment wall. Because of the fast flow over a relatively short streamwise length of the attachment-wall surface - and also because of the relatively slow diffusive transversal heat transport in laminar flows - it is reasonable to expect that the layer of the air in the jet actually influenced by the applied heating is rather thin. This heated layer is shown in Fig. 7 as the thick black line. As before, the flow regime $A$ is laminar and the jet separates at $S_{\llcorner}$. Now, however, the near-wall heated layer stays laminar - because of the lower local Reynolds number - even after its separation from the wall. The transition into turbulence is delayed and when it finally takes place, the jet is already too far from the wall to which is does not re-attach.

That the phenomena associated with presence of separation bubble as discussed above are real is supported by results of another experiment. It is author's pressure measurement on cylindrical attachment wall under the attached wall jets. A typical result obtained in the coldwall flow regime is presented in Fig. 8. The pressure at the wall is lower than atmospheric - and it is the pressure difference $\Delta \mathrm{P}[\mathrm{Pa}]$ between the wall and the atmosphere that keeps the jet attached - the higher $\Delta \mathrm{P}$, the stronger is the attachment. For easier comparisons with other similar pressure distribution measurements it was useful to nondimensionalise the measured pressure difference by conversion int pressure coefficient $C_{P}$ defined

$$
C_{P}=\frac{2 v \Delta P}{w}
$$

- where $w[\mathrm{~m} / \mathrm{s}]$ is the bulk velocity in the exit of the nozzle. In fact, it is an even more useful characterisation variable, the reduced pressure coefficient $c_{p} r / b$, the values of which are in Fig. 8 plotted as a function of the angular position $\boldsymbol{N}$ along the attachment surface.

Since it is the cold-flow case, the data in the example shown in Fig. 8 represent the closed bubble case (similar to that in Fig. 6). As the flow progresses from the regime $A$, there is in Fig. 8 a visible tendency towards the separation (i.e. the local decrease of the pressure force) which is the demonstration of the presence of the separation bubble. The extent of the bubble is (cf. Fig. 6) between the laminar separation point $S_{L}$ and the reattachment point $R$ after the transition into turbulence in T. Downstream from the bubble, there is the turbulentflow plateau (B). The attachment causing pressure force in this plateau is somewhat weaker than in the preseparation laminar regime $A$, nevertheless untl $S_{T}$ the jet is attached.

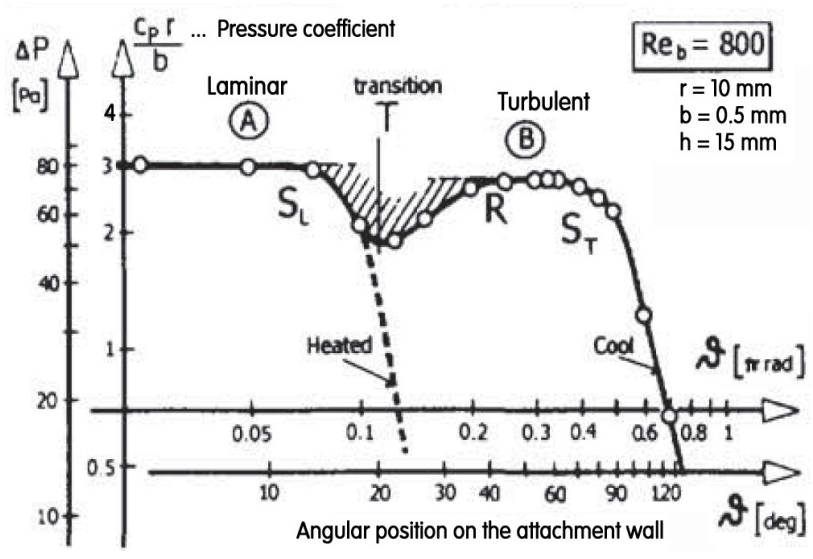

Figure 8. An example of pressure measurements on a cylindrical attachment surface. Data points reveal existence of the separation bubble in which the pressure difference, keeping the jet at the attachment wall, is decreased.

Positions of the local minima of attachment pressure difference - and their extents obtained in such measurements - as well as the character of the pressure distributions in the diagrams similar to Fig. 8 correspond very well with the explanation based on the assumed presence of the separation bubble.

\section{Transducer proposal}

The basic idea on which the discussed new electro/fluidic transducers are to be based is presented in Figs. 9 and 10 - based on the assumption of transferability of the results shown above in Fig. 4. The conditions in the nonheated regime have to be adjusted as shown in Fig. 9 so that they are sufficiently near to the separation boundary. The heating causes shifting of this boundary, Fig. 10, so that the jet separates. The mode of operation is this twopositional - ON and OFF. This is no shortcoming because the currently most popular fluidic device, 


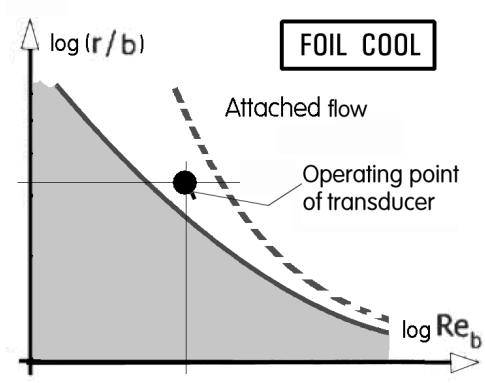

Figure 9. Principle of the proposed transducer. Parameters - the heated foil curvature radius $r$, nozzle width $b$, and inlet coldflow Reynolds number $\mathrm{Re}_{\mathbf{b}}$ indicated by the black dot symbol - result in the attached (white colour).

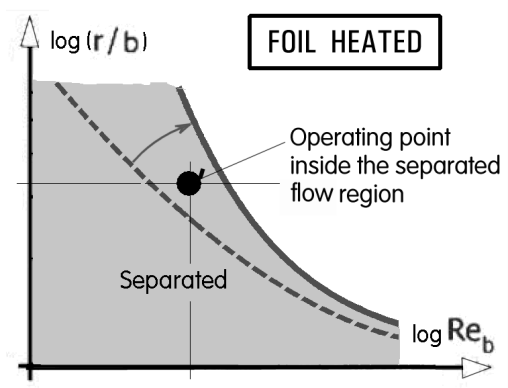

Figure 10. Proposed transducer with the foil heated. The critical boundary (full line) moved to the right and up in this diagram so that in spite of the same operating point position it is now inside the separated-flow region (shaded).

bistable diverter amplifiers, operate in the very same mode [19]. It is therefore useful to copy on the fluidic side of the transducer the symmetric configuration of such amplifiers - as is seen in Fig. 11. There is a single supply terminal, but two output terminals between which the flow is switched. Such amplifiers have two attachment walls of the opposing sides of the interaction cavity and switching the flow means.

In the proposed transducer the action of the control nozzles, the outflow from which in the configuration from Fig. 11 causes the switching of the main jet, is

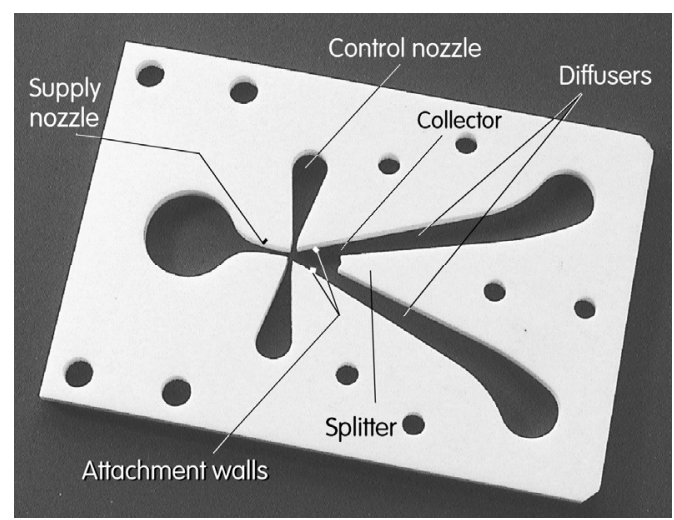

Figure 11. Photograph of the starting point of the transducer development - a typical bistable fluidic diverter amplifier.

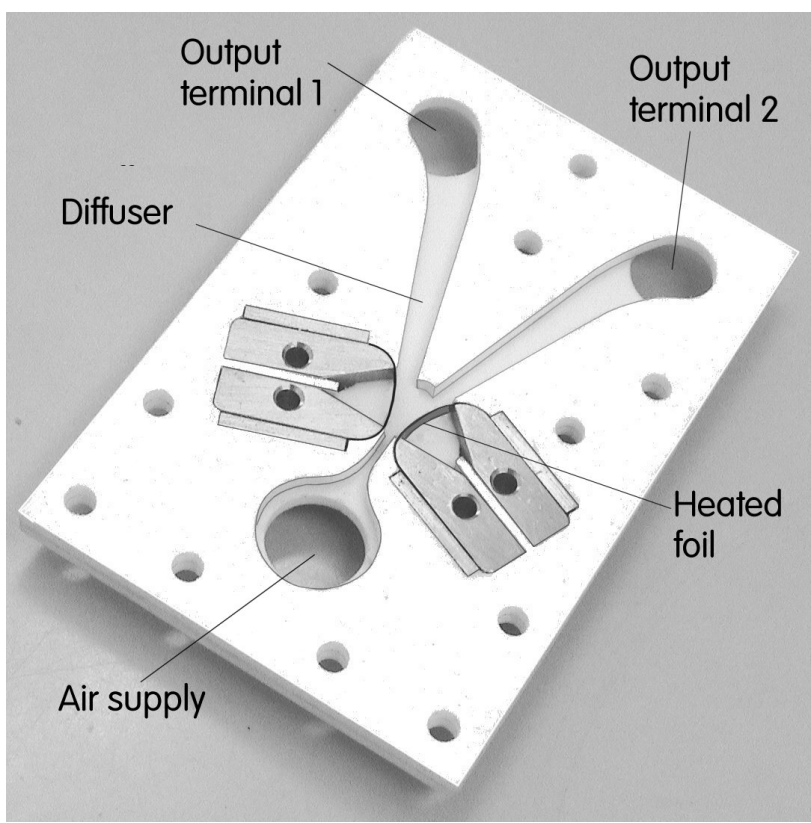

Figure 12. Photograph of the transducer model. It consist of three rectangular plates of $3 \mathrm{~mm}$ thick teflon, with cavities made by laser cutting. The design copies the usual jet-deflection Coanda-effect diverter - thus here are two heated foils, at the positions where in such diverters are the two the jet-attachment walls.

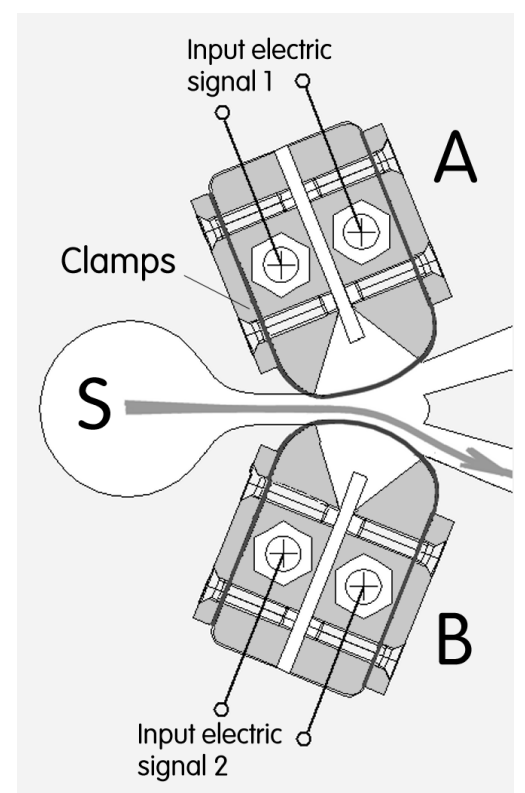

Figure 13. Drawing of the clampings of the ends of the heated foils in the massive brass components (clamps) that serve as the electric input terminals.

replaced by the action of heating on the pair of the attachment foils.

The development of the transducer at the time of writing this paper progressed to making a laboratory model.. The dimensions, as seen in Fig. 12, are larger than expected size of envisaged operational transducers but this large scale is suitable for making changes if the necessity of them arises during the tests. 


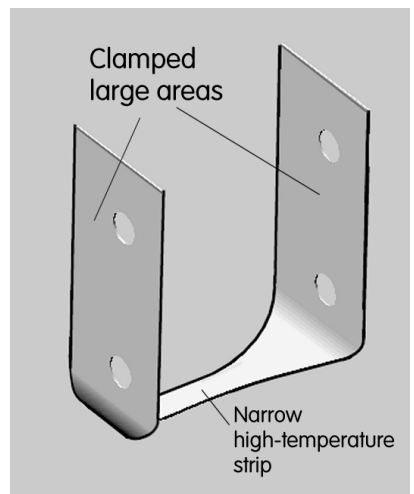

Figure 14. Spatial shaping of the foil. The central part coming into contact with the air flow is narrow to increase the local electric resistance and concentrate there the ohmic heating.

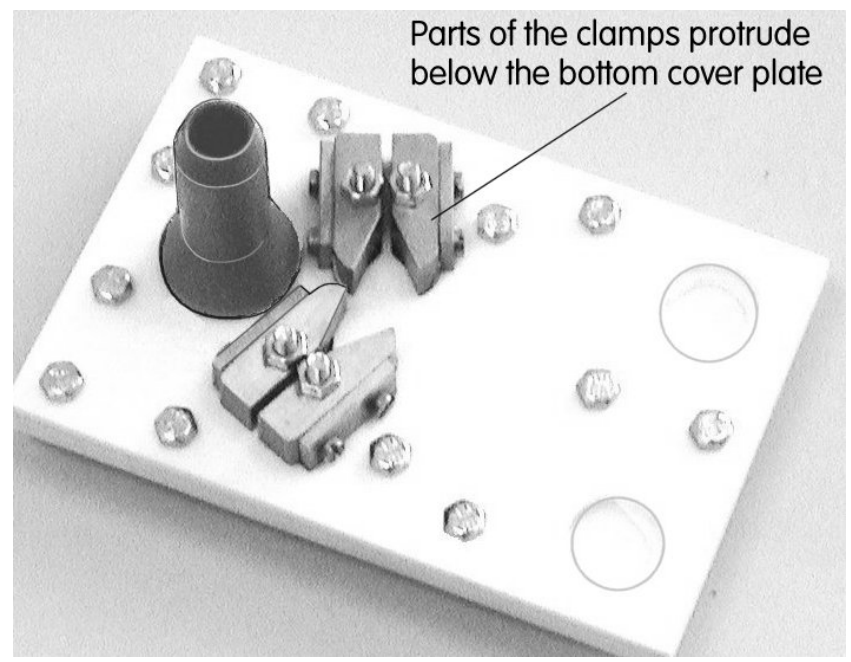

Figure 15. Photograph of the transducer model from below. The wide clamps pass through two teflon plates - while the central heated strip of the foil occupies only the main central plate, i.e. the plate which is seen from above in the disassembled transducer photograph Figure 12.

The layout of the electrical heating of the attachment foils is apparent from Figs. 12 to 16 . The thin metal foil is shaped as presented in the perspective view Fig. 16. Its . narrow central part in which the electric input current is concentrated, will be heated - while other metal parts are very massive and should therefore allow passage of quite high currents without heating.

For most of the time, it is assumed that the air jet will be attached to one of the cold foils. Let us suppose it is attached to the foil $A$ in Fig. 13. An electric current pulse delivered into the A-side foil will cause the air jet to separate and, this side being no more attractive, will switch and attach to the opposite cold foil B. It will stay attached there even when the current pulse finished. Thus the foil on the A-side shall have enough opportunity for its cooling down.

Of course, it is necessary to set up the electric circuit logic so that the next current pulse is delivered into the foil B. This will switch the air flow back, so that it then would leave the transducer into the output terminal on the A side.

Mechanical properties of the foils were found unsuitable for shaping them as shown in Fig. 14 prior to their placement into the transducer with a previously adjusted curvature radius. In particular, author was able to obtain $0.6 \mathrm{~mm}$ thin foil of titanium, a metal excellently suitable for high-temperature operation. This foil is, however, rather stiff. Thus the transducer was assembled first and the radius - as shown in Fig. 16 was subsequently evaluated from enlarged detail photographs.

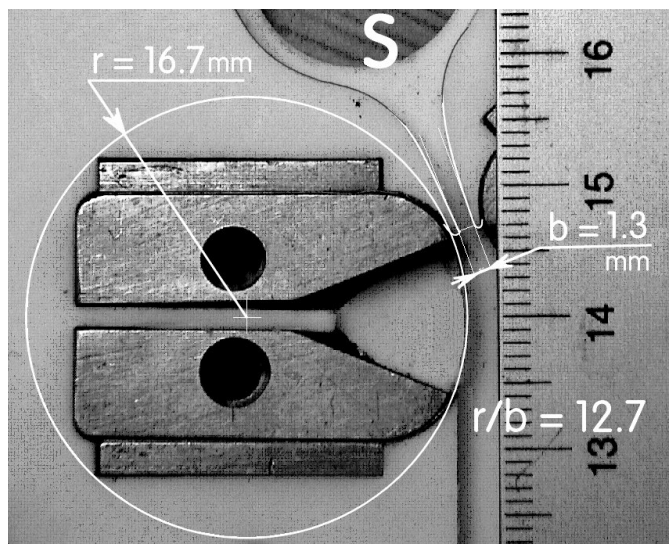

Figure 16. Detail photograph of the supply nozzle (of width b) and the foil with fitted circle to evaluate the attachment wall curvature radius $r$.

\section{Conclusions}

Author's preliminary experiments with air jet attachment to a cylindrically shaped attachment wall which has a shape of a very thin metal foil - and the separation from this foil when it is heated by electric current passing through it - show that relatively small temperature difference heating suffices to change substantially the flowfield inside the device. The idea has been developed into a transducer with characteristic features resembling a fluidic bistable diverter.

Based on the gained experience, the proposed new principle of converting electric signal into a control of fluid flows, with complete absence of movable or deformable components, seems to be quite plausible.

\section{Acknowledgements}

The development of the transducer has been supported by research grant Nr. 13-23046S obtained from GAČR the Czech Science Foundation. Author also received institutional support RVO:61388998. Author is grateful to Mr. Miroslav Pavelka for his help with experimental investigation. 


\section{References}

1. V. Tesař, Chapter 5 in Pressure Driven Microfluidics, (Artech House Publ., Boston-London, 2007)

2. V. Tesař, Chemical Engineering Research and Design 87, 181 (2009)

3. W. B. Zimmerman et al., Food Bioprod. Process, 87, 215 (2009)

4. W. M. Winslow, U.S. Patent 2,417,850, filed 1947

5. L. Zipser, L. Richter, U. Lange, Sensors and Actuators A: Physical, 92, 318 (2001)

6. V. Tesař, Czechoslovak Patent Nr. 139202, filed June 1967

7. V. Tesař, Czechoslovak Patent Nr. 134391, filed October 1966

8. Rehsteiner F.: Signal transducer for fluidic controls, US Patent 3760848 A, filed October 1971

9. S. Sugaya, S. Ando, F. Suzuki F., Fluidics Quarterly, 5, 13 (1973)

10. V. Tesař, V. Janíček, Czechoslovak Patent 159335 , filed September 1973

11. V. Tesař, Control of flow separation by temperature gradient (Proc. of Euromech Colloq. 377 Praha, Czechoslovakia May 1998)

12. V. Tesar, Flow separation controlled by wall heating - numerical solution (Proc. of 20th Internat. Conf.

Kouty nad Desnou Czech Republic, June 2001)

13. Owen P.R., Klanfer L.: On the laminar boundarylayer separation from the leading edge of a thin aerofoil, ARC Current Paper 220, 1953

14. V. Tesař, Chemical Engineering Research and Design, 87, 817 (2009)

15. V. Tesař, Chemical Engineering Journal 168, 23 (2011)

16. V. Tesař, J. Kordík J., Sensors and Actuators A: Physical, 187, 105 (2012)

17. V. Tesar̆, Chemical Engineering Research and Design, 87, 817 (2009)

18. V. Tesař, Chemical Engineering Science, 116, 843 (2014)

19. V. Tesař, H. Bandulasena, Experiments in Fluids, 50, 1225 (2011) 